\title{
Emittance dilution and halo creation during the first milliseconds after injection at the Fermilab Booster
}

\author{
Panagiotis Spentzouris* and James Amundson* \\ ${ }^{*}$ Fermi National Accelerator Laboratory, P.O. Box 500, Batavia, IL 60510, USA
}

\begin{abstract}
.
During the past year, the Fermilab Booster has been pushed to record intensities in order to satisfy the needs of the Tevatron collider and neutrino programs. This high intensity makes the study of space-charge effects and halo formation highly relevant to optimizing Booster performance. We present measurements of beam width evolution, halo formation, and coherent tune shifts, emphasizing the experimental techniques used and the calibration of the measuring devices. We also use simulations utilizing the 3D space-charge code Synergia to study the physical origins of these effects.
\end{abstract}

Keywords: space-charge, halo, parallel computing, Booster, injection PACS: $29.27 . \mathrm{Bd}, 29.27 . \mathrm{Fh}$

\section{THE SYNERGIA FRAMEWORK}

Several computer simulations of space-charge effects in circular accelerators using particle-in-cell techniques have been developed $[1,2,3]$. These simulations have emphasized the transverse dynamics while using a less rigorous approach for the longitudinal dynamics. Synergia [6] is a package for state-of-the-art simulation of linear and circular accelerators with a fully threedimensional (3D) treatment of space charge, and the capability to use arbitrary order maps for the single-particle optics modeling.

Synergia is designed to be a general-purpose tool with an interface that is accessible to accelerator physicists who are not experts in simulation. Space-charge calculations are computationally intensive, typically requiring the use of parallel computers. The implementation of Synergia utilizes Particle-In-Cell (PIC) techniques and is fully parallel, including the particle tracking and space-charge modules. The code itself is a hybrid system based on the IMPACT [4] space-charge code and the mxyzptlk/beamline libraries [5]. Synergia includes enhancements to these codes as well as new modules. A description of the Synergia framework, including details of the new capabilities can be found in Ref. [6] and at the Synergia project web pages: http: //cepa.fnal.gov/psm/aas/Advanced \ _Accelerator \_Simulation.html.

The space-charge module uses the path length along the reference trajectory, $s$, as the independent variable and implements a variety of different boundary conditions: (i) 3D open boundary conditions, (ii) open boundary conditions transversely and periodic boundary conditions longitudinally, and (iii) solvers for round and rectangular conducting pipes, where the longitudinal bound-

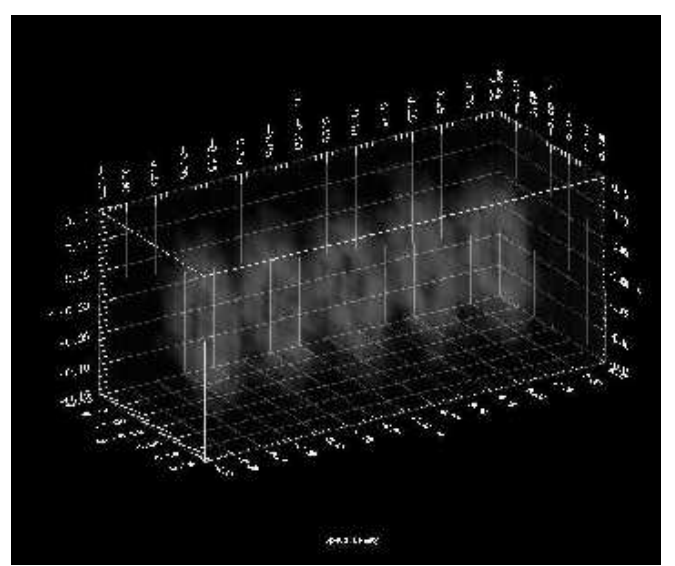

FIGURE 1. Configuration space for a simulated beam slice of the Fermilab Booster.

ary condition is either open or periodic. Synergia has multi-turn injection capabilities and can follow multiple bunches longitudinally; an example of such multi-bunch run is shown in Figure 1.

\section{Performance}

For high precision 3D simulations of realistic machines, large numbers of macroparticles, on the order of $10^{6-7}$, and fine space-charge grids, typical size of $33 \times 33 \times 257$, are required. In order to obtain the necessary computing power for such simulations, we have ported our code to different parallel machines, including commodity PC clusters with various configurations, as well as specialized parallel computers. A summary of the performance of the Synergia code for FNAL Booster 


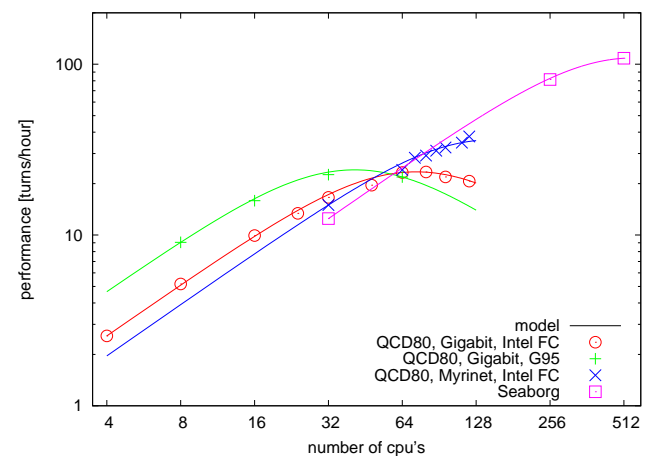

FIGURE 2. Synergia performance, measured on Booster turns per hour, versus number of processors, for different parallel machines.

modeling on some of these machines is shown in Figure 2 , as a function of the number of processors used to run the simulation. Performance and its scaling on the number of processors used depends on an interplay of networking speed, latency, and processing speed. For our Booster simulations, peak performance of $\sim 100$ Booster turns per hour is obtained running on 512 processors on the NERSC supercomputer.

\section{Benchmarking}

In order to verify the accuracy of our simulation we provide a test suite, in which we model several cases simple enough to perform comparisons with semi-analytic calculations [6]. The suite includes models which follow the evolution of a K-V beam distribution and the evolution of the second moments of a Gaussian beam distribution in idealized FODO channels; the solutions are then compared to the solution of the envelope equation. We also compare FODO channel results from Synergia with another 3D space-charge code, and tune shifts predicted by Synergia to that of the Laslett tune shift formula. In all these comparisons, the Synergia code is in good agreement with the theoretical expectations. An example of such a comparison is shown in Figure 3. Here we model a cold, uniform density, $100 \mathrm{~mA}$ proton beam, with kinetic energy of $250 \mathrm{MeV}$, in a FODO channel with rf cavities, and compare with the MaryLie/IMPACT (ML/I) [7] 3D code. The agreement between the two codes is excellent. The channel consists of two $0.15 \mathrm{~m}$ focusing quadrupoles (fquad), with a gradient of $6 \mathrm{~T} / \mathrm{m}$, a $0.30 \mathrm{~m}$ defocusing quadrupole (dquad), with $-6 \mathrm{~T} / \mathrm{m}$ gradient, four $0.10 \mathrm{~m}$ drifts (dr), and two $1 \mathrm{~m}$ rf cavities (cav), with frequency $700 \mathrm{MHz}$. The rf cavities are treated by computing the linear transfer maps, including the effects of acceleration, and using numerical integration of the map coefficients. This requires a knowledge of the on-axis electric field

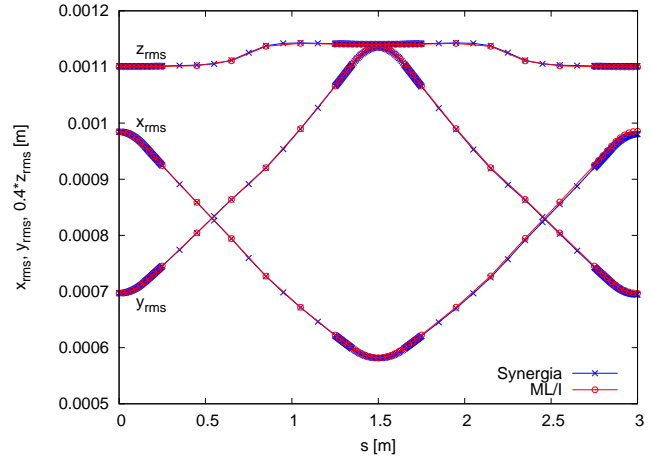

FIGURE 3. Comparison of the Synergia and MaryLie/IMPACT predictions for the r.m.s. beam envelopes of a cold beam propagating in a FODO channel with rf cavities.

and its derivative. For this example, the functional form of the field is given by $E(z)=E_{0} \cos (\omega t+\phi)$. The beamline is arranged in the following way: (fquad $\mathrm{dr}$ cav $\mathrm{dr}$ dquad dr cav dr fquad). The cavity phases have been set so that the first cavity accelerates the beam and the second decelerates it by the same amount. Since the beam is cold, the rms equations describe the problem exactly, as long as the beam remains cold and uniform, so there is a matched condition where the final envelopes are identical to the initial values. We used a $3 \mathrm{D}$ envelope matching code to find the matched beam parameters. We generated a numerical realization of the matched uniform distribution consisting of 100,000 particles. These particles were used as the input of both Synergia and ML/I.

\section{FERMILAB BOOSTER STUDIES}

The Booster [8] is a rapid-cycling, $15 \mathrm{~Hz}$, alternating gradient synchrotron with a radius of 75.47 meters. The lattice consists of 96 combined function magnets in 24 periods, with nominal horizontal and vertical tunes of 6.9 and 6.7 respectively. The Booster accelerates protons from a kinetic energy of $400 \mathrm{MeV}$ to $8 \mathrm{GeV}$, at harmonic number $h=84$, using $17 \mathrm{RF}$ cavities with frequency that slews from $37.7 \mathrm{MHz}$ at injection to $52.8 \mathrm{MHz}$ at extraction. The nominal average current at injection is $\sim 42 \mathrm{~mA}$. Typically, the injection process lasts for ten Booster turns. The injected beam is a stream of bunches equally spaced at the linac RF frequency of 201.2 MHz.

There are many factors affecting the behavior of the Booster beam, including the energy spread and emittance of the injected beam, nonlinear field errors and spacecharge effects. The space-charge effects have long been believed to be responsible for a significant fraction of the observed losses in the Booster during the first 2 $\mathrm{ms}$ of the cycle (the injection, capture, and bunching phases). In this section we study how these effects affect 
both the core of the beam (envelope tune shifts) and the shape of the distribution (halo), using both Synergia and experimental data. For all the simulations we used an idealized "bare" Booster lattice without any non-linear elements, but we do employ second order maps and we use a beam with realistic energy spread, thus chromatic effects are included.

\section{Measurement of coherent tune dependence on space-charge}

We performed a study of the coherent tune in the Booster by measuring beam transmission versus machine tune, for different beam intensities. We used a coasting beam with the machine operating DC (no ramping magnets). The beam transmission was determined by measuring the beam current at injection and at 2000 turns after injection. We took data for three different values of the injected beam current 1, 3, and 9 injected Linac turns (approximately 0.042, 0.126, and 0.378 Amps respectively). For each value of the beam current we measured the transmission for different values of the machine tune, obtained by varying the corrector quadrupoles in the Booster. We varied the tune up and down from the nominal until we excited a resonance (i.e reached zero transmission). We then fit the transmission versus tune curves to obtain the location and width of the resonance. The relationship between quadrupole current and beam tune was obtained for the case of one Linac turn injected, by using a spectrum analyzer. By tracking the change in the position of the resonance and subtracting for the known tune shift due to the change in the quadrupoles, we can extract the tune shift due to space charge. In the simulation, we performed an FFT of the beam envelope to extract the coherent tune. Figure 4 shows a comparison of the measured and simulated tune shifts versus the number of turns of injected Linac beam, and the resonance widths. The results are in excellent agreement.

\section{Beam Halo}

In order to study beam halo generation we need to use a detector which provides non-destructive beam shape measurement on a turn-by-turn basis. The Booster Ionization Profile Monitor (IPM) [9] is the only such device in the Booster, capable of measuring beam profiles with a time resolution of a single turn (2.2 micro seconds at injection) at all times in the cycle. The IPM measures profiles using ions produced by the interaction of the beam with the imperfect vacuum of the machine. An applied transverse clearing field causes the ions to drift to a micro-channel plate (MCP). Since the charge of the
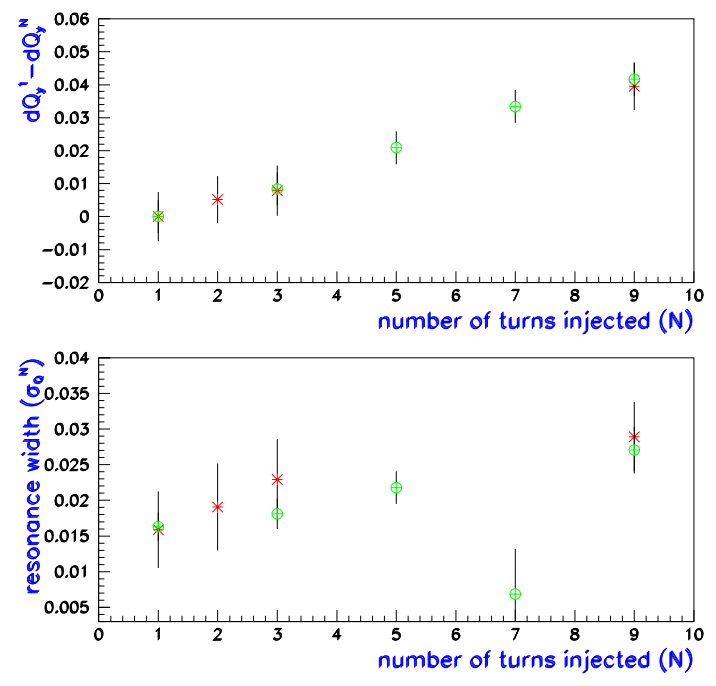

FIGURE 4. Coherent tune shifts (top) and resonance width (bottom) as a function of number of turns of beam injected. In both cases the red crosses are the measurement and the green circles are the results of the simulation.

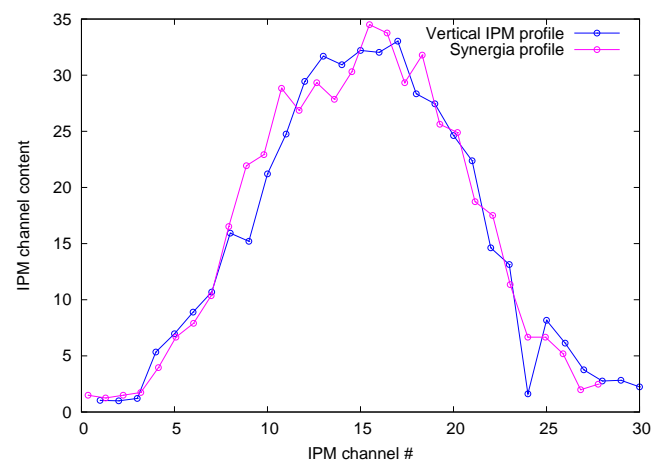

FIGURE 5. Beam profile observed in the IPM (blue line) compared to a profile generated from the Synergia simulated beam, and smeared with the IPM response model.

beam also generates a field, it affects the motion of the ions. This effect depends on the charge and the size of the beam, thus the response of the IPM must be calibrated as a function of the injected number of protons. We determined the calibration of the IPM using a detailed model of the detector constrained by independent beam profile measurements [10]. These measurements were obtained at injection, using a single wire, and at extraction, using a multiwire proportional chamber in one of the extraction lines. The calibration can be used either as a function which corrects the measured beam width or as a smearing kernel, which can be used to add the smearing effects of the IPM response to a simulated beam distribution. In Figure 5 we compare a typical beam profile observed in the IPM, with a simulated beam profile, where the beam distribution modeled by Synergia is smeared according 


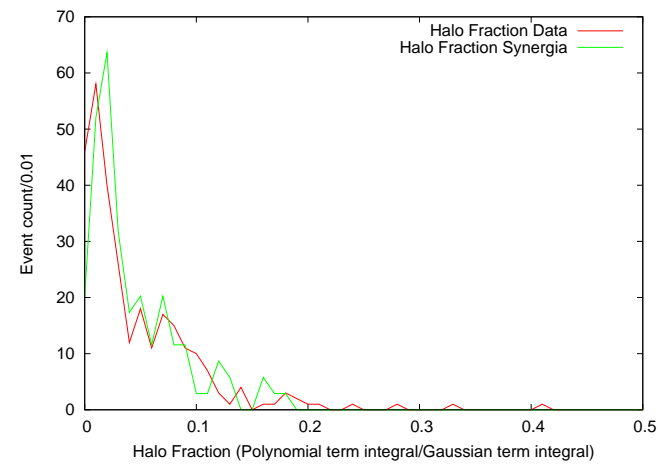

FIGURE 6. Histogram of the $L / G$ measured with the IPM (red) in the first 100 Booster turns after injection compared to the simulated $L / G$ (green).

to the IPM response model. The agreement is excellent, thus we feel confident in using the shape of the beam distribution observed in the IPM to quantify halo generation.

One of the most commonly used quantitative measure of halo formation, based on beam shape, is the kurtosis $k \equiv \frac{\left\langle(x-\langle x\rangle)^{4}\right\rangle}{\left\langle(x-\langle x\rangle)^{2}\right\rangle^{2}}-3 .{ }^{1}$ Indeed, the simulation shows that in the presence of space-charge and beam mismatch, an initially Gaussian beam becomes leptokurtic $(k>0)$, an indication of halo. Unfortunately, the effects of the IPM smearing changes the distribution to platykurtic, thus a positive kurtosis is not an unambiguous measure of halo in our case. Instead, we employ an empirical procedure: we fit each beam profile to a function that consists of a Gaussian sitting on top of a linear background. For a simple model of halo, we ascribe $G \equiv \int$ (Gaussian part) to the core and $L \equiv \int$ (linear part) to the halo. To quantify the size of the halo we form the ration $L / G$ and monitor it through the Booster cycle. In the data, for the first 100 turns after injection, we measure an average $L / G=0.049 \pm 0.001$, while in the simulation, which includes IPM smearing effects, we extract an average $L / G=0.044 \pm 0.006$. In Figure 6 we compare a histogram of the measured $L / G$ extracted turn-by-turn for the first 100 turns after injection, with the simulated $L / G$. The agreement is very good. The simulation modeled multi-turn injection and the initial beam used in the simulation was a 6-dimensional Gaussian distribution, with the transverse beam width fixed to match the IPM measurement at injection. The horizontal and vertical rms. emittance was $3.05 \times 10^{-6} \mathrm{~m}$ rad. The momentum spread, $\Delta p / p$, was 0.0003 , and we employed second order maps for the single particle optics. We used 96 spacecharge kicks per turn, calculated on a $33 \times 33 \times 257$ com-

\footnotetext{
${ }^{1}$ A Gaussian beam distribution has $k=0$.
}

putational grid with an average of four particles per grid cell. The total beam current was 0.420 Amps. In order to match the observed $L / G$ we had to use a $20 \%$ mismatched input beam, where the transverse emittance is kept to its nominal value of $3.05 \times 10^{-6} \mathrm{~m}$ rad for both planes, but the conjugate momentum distribution is modified from the matched solution. Both space charge and chromatic effects are required to be present in the simulation in order to obtain agreement with the data.

\section{Emittance dilution}

Here we investigate how space-charge and chromatic effects affect the emittance of the Booster. In Figure 7 we plot the normalized 4-D transverse emittance ${ }^{2}$ for five different initial beam conditions, described in the caption of the figure. As expected, in the cases where the beam was matched there is no emittance growth. That is the case for both zero and non-zero momentum spread, and with the inclusion of space charge. (Our matching procedure takes into account space-charge effects on the second moments of the beam). In the mismatched cases, where the mismatch is $20 \%$, we observe a $12 \%$ increase of the beam emittance during the first 10 to 15 turns after injection. The effect is a combination of chromatic and space-charge effects and it is very similar for both the single- and multi-turn injection cases. The total current is the same, $0.420 \mathrm{Amps}$, in both cases.

\section{Filamentation of longitudinal phase-space}

Finally, we present an interesting effect we observed in the longitudinal phase-space evolution during our Booster simulations. In simulations where the model included space-charge effects and realistic momentum spread of 0.0003 , we observed an apparent coupling of neighboring longitudinal phase-space filaments ${ }^{3}$. The injected beam was bunched (200 MHz Linac rf) and it debunched very quickly as it coasted freely. In $\sim 15$ turns the filaments originated from the injected bunches were almost parallel, separated by equal momentum gaps, and there was no indication of any frequency content in the Fourier transform of the phase distribution (see Figure 8). As the distance of the filaments to their neighbors shrunk, in $\sim 10$ more turns, phase-space "bubbles" formed (see Figure 9), and the frequency content of the phase distribution showed a strong component of $200 \mathrm{MHz}$ harmon-

\footnotetext{
${ }^{2}$ Defi ned as the square root of the determinant of the covariance matrix of the transverse phase space.

${ }^{3}$ Note that we use round conductive pipe transverse boundary conditions.
} 


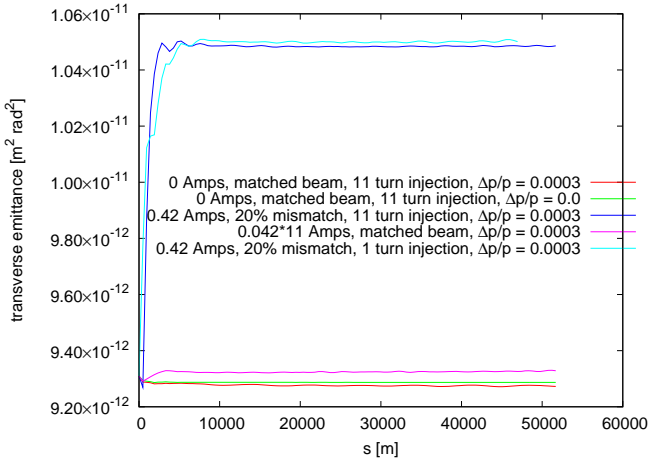

FIGURE 7. Normalized 4-D transverse emittance in $\mathrm{m}^{2} \mathrm{rad}^{2}$ for different initial conditions. The red and green curves correspond to a matched beam, with space-charge effects turned off (0 Amps) with and without a momentum spread of 0.0003 , respectively. The purple and light blue curves correspond to a beam of 0.420 Amps total current and momentum spread of 0.0003 , matched and mismatched respectively. Multi-turn injection of 11 turns is used in all of the above cases. The dark blue curve corresponds to a single turn injection simulation of a 0.420 Amp mismatched beam with 0.0003 momentum spread.

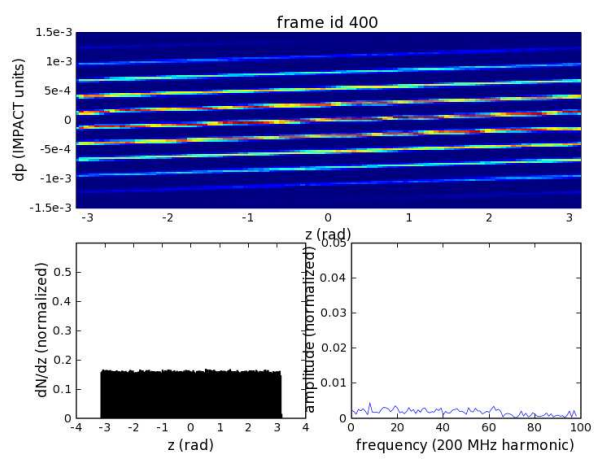

FIGURE 8. Longitudinal phase-space after 16 turns. Top plot $\Delta E$ versus phase. Bottom left beam phase distribution, and right its Fourier transform.

ics. The effect does not appear to be an artifact of the statistics used in the simulation, as we observed the exact same behavior with two and four times the original statistics of $1 \mathrm{M}$ macroparticles. It also did not change when we changed the size of the computational grid.

Although such behavior is not documented under controlled machine operation, there is evidence during normal machine running that the " $200 \mathrm{MHz}$ linac structure persists" late in the machine cycle. We have requested machine studies during the next DC operation of the Booster to further study this effect. A discussion of the origins of such an effect is presented in Reference [11]. We are in the process of investigating the variation of the effect observed in our simulations with the initial condi-

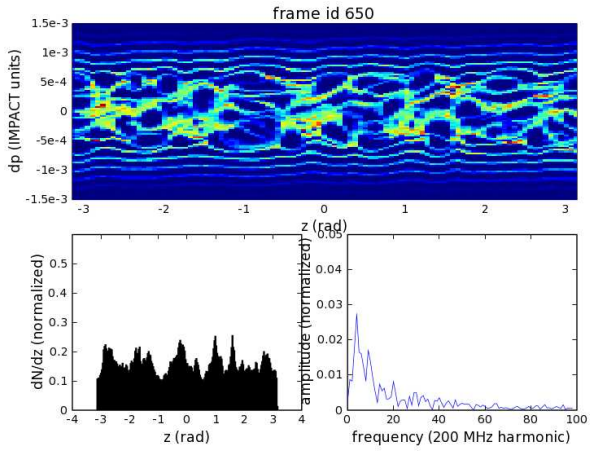

FIGURE 9. Longitudinal phase-space after 27 turns. Top plot $\Delta E$ versus phase. Bottom left beam phase distribution, and right its Fourier transform.

tions of the simulations according to the above discussion.

\section{ACKNOWLEDGMENTS}

This work was performed under the auspices of a Scientific Discovery through Advanced Computing project, "Advanced Computing for $21^{\text {st }}$ Century Accelerator Science and Technology", which is supported by the US DOE/SC Office of High Energy Physics and the Office of Advanced Scientific Computing Research.

\section{REFERENCES}

1. F. Jones, G. H. Mackenzie, and H. Schonauer, Part. Accel. 31, 199 (1990).

2. S. Machida, in Computational Accelerator Physics, edited by R. Ryne, AIP Conf. Proc. No. 297 (AIP, New York, 1994), p. 459.

3. J. Galambos, J. Holmes, D. Olsen, A. Luccio, and J. Beebe-Wang, ORBIT User's Manual, Oak Ridge National Laboratory, SNS/ORNL/AP Technical Note No. 011, 1999.

4. J. Qiang, R. D. Ryne, S. Habib and V. Decyk, J. Comp. Phys. 163, 434 (2000).

5. L. Michelotti, FERMILAB-CONF-91-159

6. J. Amundson, P. Spentzouris, J. Qiang, R. Ryne, FNALPub-04/136-CD, submitted to PRSTAB

7. http://scidac.nersc.gov/accelerator/ $\mathrm{mli} / \mathrm{manual} \cdot \mathrm{pdf}$

8. Booster Staff 1973 Booster Synchrotron ed E L Hubbard Fermi National Accelerator Laboratory Technical Memo $T M-405$

9. J. Zagel, D Chen, J. Crisp, 1994 Beam Instrumentation Workshop, AIP Conference Proceedings 333, pp384-390.

10. J. Amundson et al, Phys. Rev. ST Accel. Beams 6, 102801 (2003).

11. I. Hofmann, Part. Accel. 34, 211 (1990) 